\title{
“Conocimiento" en un Sistema de Drones Levantando una Carga
}

\author{
Joel Suárez Cansino a,*, Virgilio López Morales, César Eduardo Juárez Vargas \\ ${ }^{a}$ Cuerpo Académico de Computación Inteligente- Área Académica de Computación y Electrónica \\ Instituto de Ciencias Básicas e Ingeniería - Universidad Autónoma del Estado de Hidalgo \\ Carretera Pachuca-Tulancingo Km. 4.5, Colonia Carboneras, CP 42184, Mineral de la Reforma, Hidalgo, México
}

\begin{abstract}
Resumen
El sistema de ecuaciones diferenciales asociado al problema de levantar y transportar una carga por medio de un grupo de drones tiene una estructura compleja que presenta acoplamiento, y una serie de transformaciones lineales y no lineales sobre diversas variables dinámicas. Esencialmente, se observan transformaciones del tipo rotacional (a través de la presencia de matrices skew-symmetric), traslacional (transformación afín) y proyectivas (por medio de matrices de rotación de sistemas de coordenadas locales); lo que permite lanzar el ansage de que el "conocimiento" (entendido como información puesta en acción) que un sistema tiene se hace patente por medio de la manipulación de información a través de transformaciones lineales o no lineales. El sistema de drones que transportan una carga resulta interesante porque, además de lo anteriormente escrito, se presta para introducir la idea de "intencionalidad" del sistema para lograr un objetivo, y de que ésta también tenga una interpretación como transformación. Este trabajo introduce los primeros pasos en el estudio del concepto de "conocimiento" en esta dirección. En este sentido, el giro interpretativo (basado en la idea de pensamiento lateral) que se le da a la dinámica del sistema es único y prometedor.
\end{abstract}

Palabras Clave: Sistema Dinámico, Dron, Energías Cinética y Potencial, Sistema Universal y Local de Coordenadas, Sistema de Ecuaciones Diferenciales, Centro de Masa, Transformación Lineal, Cambio de Base

\section{Introducción}

El análisis formal, por medio de la mecánica clásica, de un sistema compuesto de muchos cuerpos que interactúan entre sí, es un problema bastante conocido en física. Las cosas resultan mucho más interesantes cuando las componentes del sistema tienen características inteligentes, como es el caso de un conjunto de hormigas transportando un gusano. Es sorprendente la forma en que estos insectos colaboran en sincronía para lograr su objetivo.

¿Qué aspectos se necesitan considerar en el caso en que los elementos del sistema tengan que volar, en lugar de trasladarse por tierra, para transportar un objeto? Nótese que un sistema físico solamente toma en cuenta interacciones entre partículas que se mueven aleatoriamente. Sin embargo, el análisis del sistema que se propone es más complicado ya que, además de lo que el sistema físico hace, se incluye intencionalidad. Más allá de la posible utilidad aplicativa que tiene el analizar e implementar un sistema con estas características, que la hay, este

\footnotetext{
${ }^{*}$ Autor en correspondencia.

Correo electrónico: jsuarez@uaeh.edu.mx (Joel Suárez Cansino)

URL: www . uaeh.edu .mx (Joel Suárez Cansino)
}

artículo tiene el interés de hacer algunos comentarios interesantes acerca de la descripción formal del mismo.

Lo que se considera aquí es que el conocimiento es un proceso encargado de accionar información; es decir, conocimiento es información puesta en acción. Para ello, se requieren transformaciones que, recibiendo a la información como argumento transformen a ésta de cierta forma, para producir más información, y así sucesivamente. El concepto clave es el de transformación.

¿A qué tipo de objetos se aplica una transformación? Ya se ha escrito que estos objetos representan información, entendida ésta como un conjunto de datos que describen algo; pero, ¿qué cosa es un dato? La respuesta común es que un dato es el valor que adquiere una variable de interés en la descripción de un sistema. Pero entonces se llega a cuestionar el significado de variable e incluso de sistema. Se dirá que una variable es una característica de un sistema que es útil para describir aspectos importantes del mismo, entendiendo que un sistema está definido por un conjunto de componentes que interactúan entre sí para lograr un propósito. Así, por ejemplo, el sistema definido por una masa sujetada por una cuerda que, a su vez, se encuentra fija por el otro extremo en un punto de algún techo, tiene como variable al ángulo $\theta$ que se forma entre la vertical 
que pasa por el punto de sujeción en el techo y la cuerda, en algún instante de tiempo $t$.

Si la masa del péndulo simple se suelta, y no existe otra fuerza externa más que la gravedad y la fuerza de tensión de la cuerda sobre la masa, entonces la masa empieza a oscilar y la posición de la misma al tiempo $t$ es dada por $\theta(t)$, la cual representa un dato. Por supuesto, puede no ser suficiente considerar esta variable solamente para una completa descripción del sistema y que se requiera introducir otra variable, por ejemplo la velocidad angular $\omega$. Por consiguiente, al tiempo $t$ se tendrán dos datos, uno correspondiente a la posición angular $\theta(t)$ y otro asociado a la velocidad angular $\omega(t)$, lo cual determina la información $(\theta(t), \omega(t))$ en ese instante.

El origen de los datos puede ser diverso, en lugar de lo anterior, se puede decir que la posición $x$ de un objeto es la variable de interés, por lo que su valor a un tiempo $t, x(t)$, es un dato importante. En el caso tridimensional se tendrá la información $\boldsymbol{x}=(x(t), y(t), z(t))$, la cual es un vector. Si se habla de accionar la información, entonces $T(x, y, z)$ indica que la transformación $T$ actúa sobre $\boldsymbol{x}$, convirtiéndolo en $T(x, y, z)$, que es nueva información. La situación puede volverse más compleja si más de una transformación está involucrada.

¿Cómo se relaciona esto con el estudio de interés planteado al principio de la introducción? Las siguientes secciones del artículo se orientan a comentar sobre la naturaleza de los datos, la estructura de las transformaciones que actúan sobre ellos y las relaciones matemáticas que guardan las variables entre sí, así como la conformación de información.

\section{Sistemas de Coordenadas}

Para empezar, considérese que los datos provienen del hecho de que existe interés en ubicar en el espacio un objeto que se encuentra inmerso en él. La fuente de los datos es entonces bien el espacio Euclideano bidimensional, o bien el espacio Euclideano tridimensional. Puesto que el primero está inmerso en el segundo, se puede suponer que los datos provienen de este último; es decir, la información acerca de un objeto inmerso en el espacio es dada por la posición del mismo al tiempo $t$; a saber, $\boldsymbol{x}(t)$. Sin embargo, los valores asociados a las componentes de $\boldsymbol{x}$ dependen mucho del punto en el espacio desde el que se miden éstas.

\subsection{Sistema Universal de Coordenadas}

Para evitar la relatividad de la información acerca de la posición de un objeto en el espacio, se maneja la idea de Sistema Universal de Coordenadas. Una característica esencial de este sistema es que es inercial y, para simplificar, tiene velocidad constante nula. Toda información sobre coordenadas de un objeto se hace en relación con el origen de este sistema de coordenadas, a menos que se diga otra cosa.

Con respecto al punto origen del Sistema Universal de Coordenadas, se define un conjunto de tres vectores que es generador de los elementos del espacio. Puesto que este conjunto generador es mínimo, se dice que el conjunto es una base del espacio tridimensional, y se denota como $\boldsymbol{\beta}=\left\{\boldsymbol{\beta}_{1}, \boldsymbol{\beta}_{2}, \boldsymbol{\beta}_{3}\right\}$. Todo vector $\boldsymbol{x}$ en el espacio se puede escribir entonces como una combinación lineal de los elementos del conjunto generador; es decir, $\boldsymbol{x}=a_{1} \boldsymbol{\beta}_{1}+a_{2} \boldsymbol{\beta}_{2}+a_{3} \boldsymbol{\beta}_{3}$, lo cual en términos matriciales se escribe como (Hoffman and Kunze, 1971)

$$
[\boldsymbol{x}]_{\beta}=\left[\begin{array}{l}
a_{1} \\
a_{2} \\
a_{3}
\end{array}\right]_{\beta}
$$

siendo $[\boldsymbol{x}]_{\boldsymbol{\beta}}$ la matriz de coordenadas del vector $\boldsymbol{x}$ en la base ßק. ¡Pero momento...! ¿Con respecto a qué base se determinan las componentes de los elementos de la base $\beta$ ? Una respuesta inmediata sería: pues con respecto a la base $\beta$ misma. En este caso,

$$
\left[\boldsymbol{\beta}_{1}\right]_{\boldsymbol{\beta}}=\left[\begin{array}{l}
1 \\
0 \\
0
\end{array}\right]_{\boldsymbol{\beta}},\left[\boldsymbol{\beta}_{2}\right]_{\boldsymbol{\beta}}=\left[\begin{array}{l}
0 \\
1 \\
0
\end{array}\right]_{\boldsymbol{\beta}} \mathrm{y}\left[\boldsymbol{\beta}_{3}\right]_{\boldsymbol{\beta}}=\left[\begin{array}{l}
0 \\
0 \\
1
\end{array}\right]_{\boldsymbol{\beta}}
$$

pero esta no es la forma usual en la que se definen los elementos de una base. En la sombra, se emplea una base especial del espacio tridimensional denominada base canónica; a saber,

$$
\boldsymbol{\beta}_{c}=\{(1,0,0),(0,1,0),(0,0,1)\}
$$

por lo que

$$
\left[\boldsymbol{\beta}_{1}\right]_{\boldsymbol{\beta}_{c}}=\left[\begin{array}{l}
\beta_{11} \\
\beta_{12} \\
\beta_{13}
\end{array}\right]_{\boldsymbol{\beta}_{c}},\left[\boldsymbol{\beta}_{2}\right]_{\boldsymbol{\beta}_{c}}=\left[\begin{array}{l}
\beta_{21} \\
\beta_{22} \\
\beta_{23}
\end{array}\right]_{\boldsymbol{\beta}_{c}} \text { y }\left[\boldsymbol{\beta}_{3}\right]_{\boldsymbol{\beta}_{c}}=\left[\begin{array}{l}
\beta_{31} \\
\beta_{32} \\
\beta_{33}
\end{array}\right]_{\boldsymbol{\beta}_{c}}
$$

o equivalentemente,

$$
\boldsymbol{\beta}=\left\{\left(\beta_{11}, \beta_{12}, \beta_{13}\right),\left(\beta_{21}, \beta_{22}, \beta_{23}\right),\left(\beta_{31}, \beta_{32}, \beta_{33}\right)\right\}
$$

Por todo lo anterior, se concluye que el espacio tridimensional cuenta con muchas bases posibles, cuyos elementos se definen en relación con la base canónica.

\subsubsection{Transformaciones}

Es bien sabido que conjuntos de puntos en una región del espacio se pueden estirar, encoger, reflejar, doblar, etc., de tal forma que aparezcan posteriormente en otra región del mismo espacio. Este es un comportamiento usual de las soluciones de algunos sistemas dinámicos.

Si $\boldsymbol{x}$ es un punto en el espacio que contiene información, entonces $T(\boldsymbol{x})$ dice que se opera sobre esta información para obtener más información. $T$ se conoce como transformación, y ayuda a accionar la información en el proceso de conocer o manifestar conocimiento ( $¿$ o es mejor denominar a $T$ trinsformación?).

La teoría elemental de las transformaciones es más general y considera elementos de un espacio Euclideano de $n$ dimensiones en el dominio de la transformación, mientras que el contradominio consiste de un espacio de dimensión $m$, situación que se denota como $T: \mathbb{R}^{n} \rightarrow \mathbb{R}^{m}$, siendo $\mathbb{R}$ los reales. Así, un punto $\boldsymbol{x} \in \mathbb{R}^{n}$ tiene $n$ componentes y se expresa como la $n$-upla $\boldsymbol{x}=\left(x_{1}, x_{2}, \ldots, x_{n}\right)$. Similarmente, el punto $\boldsymbol{x}$ transformado, $T(\boldsymbol{x})$, es un elemento de $\mathbb{R}^{m}$, por lo que se expresa como $T(\boldsymbol{x})=\boldsymbol{y}$, siendo $\boldsymbol{y}=\left(y_{1}, y_{2}, \ldots, y_{m}\right)$. 
Puesto que los espacios $\mathbb{R}^{n}$ y $\mathbb{R}^{m}$ son en general diferentes, entonces sus bases tienen estructura diferente, aunque siguen siendo ordenadas. Si $\boldsymbol{\beta}$ y $\boldsymbol{\beta}^{\prime}$ denotan, respectivamente, bases de $\mathbb{R}^{n}$ y $\mathbb{R}^{m}$, y $\boldsymbol{\beta}_{c}, \boldsymbol{\beta}_{c}^{\prime}$ sus correspondientes bases canónicas, entonces $\boldsymbol{\beta}=\left\{\boldsymbol{\beta}_{1}, \boldsymbol{\beta}_{2}, \ldots, \boldsymbol{\beta}_{n}\right\}, \boldsymbol{\beta}^{\prime}=\left\{\boldsymbol{\beta}_{1}^{\prime}, \boldsymbol{\beta}_{2}^{\prime}, \ldots, \boldsymbol{\beta}_{m}^{\prime}\right\}$, siendo $\boldsymbol{\beta}_{i}$ y $\boldsymbol{\beta}_{j}^{\prime}$ expresados en base canónica.

Al igual que en el caso tridimensional, existen varias bases para cada uno de los espacios $\mathbb{R}^{n}$ y $\mathbb{R}^{m}$, cuyos elementos se expresan en términos de las correspondientes bases canónicas. Nuevamente, ¡la base canónica en la sombra! De aquí, surge la cuestión de conocer la forma en que la transformación $T$ queda afectada cuando, en lugar de usar la base $\beta$ para representar la información $\boldsymbol{x}$ se usa la base $\boldsymbol{\beta}_{1}$ y en lugar de usar la base $\boldsymbol{\beta}^{\prime}$ para representar $T(\boldsymbol{x})$ se usa la base $\boldsymbol{\beta}_{1}^{\prime}$. Si la transformación es lineal, entonces se le puede asociar una matriz $[T]_{\beta \beta^{\prime}}$ que funciona de manera similar a como lo hace $T$ sobre la información $\boldsymbol{x}$, sólo que en lugar de $T(\boldsymbol{x})$ se tiene $[T]_{\boldsymbol{\beta} \boldsymbol{\beta}^{\prime}}[\boldsymbol{x}]_{\boldsymbol{\beta}}$. El contenido de la matriz $[T]_{\boldsymbol{\beta} \boldsymbol{\beta}^{\prime}}$ depende de las bases $\boldsymbol{\beta}$ y $\boldsymbol{\beta}^{\prime}$ utilizadas para el dominio y el contradominio, respectivamente. Puesto que la matriz $[T]_{\beta \beta^{\prime}}$ tiene tamaño $m \times n$ y la matriz $[\boldsymbol{x}]_{\beta}$ tiene tamaño $n \times 1$, entonces el producto $[T]_{\beta \beta^{\prime}}[x]_{\beta}$ está bien definido y tiene tamaño $m \times 1$.

Supóngase que existe una matriz $[P]_{\boldsymbol{\beta}_{1} \boldsymbol{\beta}}$ que transforma un vector en base $\beta_{1}$ a un vector en base $\beta$; es decir,

$$
[P]_{\beta_{1} \beta}[x]_{\beta_{1}}=[x]_{\beta}
$$

y ocurre algo similar en el contradominio; en otras palabras, $[Q]_{\boldsymbol{\beta}_{1}^{\prime} \boldsymbol{\beta}^{\prime}}[\boldsymbol{y}]_{\boldsymbol{\beta}_{1}^{\prime}}=[\boldsymbol{y}]_{\boldsymbol{\beta}^{\prime}}$. Puesto que $[T]_{\boldsymbol{\beta} \boldsymbol{\beta}^{\prime}}[\boldsymbol{x}]_{\boldsymbol{\beta}}=[\boldsymbol{y}]_{\boldsymbol{\beta}^{\prime}}$, se sigue que

$$
[T]_{\boldsymbol{\beta} \boldsymbol{\beta}^{\prime}}[P]_{\boldsymbol{\beta}_{1} \boldsymbol{\beta}}[\boldsymbol{x}]_{\boldsymbol{\beta}_{1}}=[Q]_{\boldsymbol{\beta}_{1}^{\prime} \boldsymbol{\beta}^{\prime}}[\boldsymbol{y}]_{\boldsymbol{\beta}_{1}^{\prime}}
$$

o bien

$$
[Q]_{\boldsymbol{\beta}_{1}^{\prime} \boldsymbol{\beta}^{\prime}}^{-1}[T]_{\beta \beta^{\prime}}[P]_{\beta_{1} \beta}[\boldsymbol{x}]_{\boldsymbol{\beta}_{1}}=[\boldsymbol{y}]_{\boldsymbol{\beta}_{1}^{\prime}}
$$

o equivalentemente,

$$
[Q]_{\beta^{\prime} \beta_{1}^{\prime}}[T]_{\beta \beta^{\prime}}[P]_{\beta_{1} \beta}[x]_{\beta_{1}}=[y]_{\beta_{1}^{\prime}}
$$

Es decir, la matriz $[Q]_{\boldsymbol{\beta}^{\prime} \boldsymbol{\beta}_{1}^{\prime}}[T]_{\boldsymbol{\beta} \boldsymbol{\beta}^{\prime}}[P]_{\boldsymbol{\beta}_{1} \boldsymbol{\beta}}$, definida en términos de la matriz de la transformación $T$ en las bases viejas del dominio y el contradominio, toma información del dominio en términos de la base nueva y la asocia con información del contradominio en términos de la base nueva. ¡Recorcholis! Esta transformación tiene como dominio y contradominio a los mismos espacios que tiene la transformación $T$.

¿Será que esta transformación es la mismísima $T$ pero más revolcada?¿Cómo se interpreta esto en términos de información accionada; es decir, de conocimiento? Para responder estas preguntas, sea

$$
[T]_{\boldsymbol{\beta}_{1} \boldsymbol{\beta}_{1}^{\prime}}=[Q]_{\boldsymbol{\beta}^{\prime} \boldsymbol{\beta}_{1}^{\prime}}[T]_{\boldsymbol{\beta} \boldsymbol{\beta}^{\prime}}[P]_{\boldsymbol{\beta}_{1} \boldsymbol{\beta}}
$$

la matriz de la transformación $T$ respecto a las bases nuevas.

Lo que sigue a continuación es aplicar un poco más profundamente en este tema técnicas de pensamiento lateral que Edward de Bono sugiere (de Bono, 2009). ¿Son los puntos $[\boldsymbol{x}]_{\beta} \mathrm{y}$ $[\boldsymbol{x}]_{\boldsymbol{\beta}_{1}}$ diferentes; es decir, representan diferente información en el dominio de $T$ ? ¿Qué con $[\boldsymbol{y}]_{\boldsymbol{\beta}^{\prime}}$ y $[\boldsymbol{y}]_{\boldsymbol{\beta}_{1}^{\prime}}$ en el contradominio?
Las respuestas a estas preguntas están dadas en términos de las correspondientes bases canónicas, como sombras de cualesquiera bases para el dominio y contradominio de la transformación $T$; a saber, $\boldsymbol{\beta}_{c}$ y $\boldsymbol{\beta}_{c}^{\prime}$.

Así, se puede demostrar que $[\boldsymbol{x}]_{\boldsymbol{\beta}}$ y $[\boldsymbol{x}]_{\boldsymbol{\beta}_{1}}$ no son otra cosa que codificaciones diferentes de la misma información $[\boldsymbol{x}]_{\boldsymbol{\beta}_{\boldsymbol{c}}}$. Sólo que, en lugar de codificar explícitamente desde la sombra, se codifica implícitamente sobre la sombra a partir de la matriz $[P]_{\boldsymbol{\beta}_{1} \boldsymbol{\beta}}$ o bien $[P]_{\boldsymbol{\beta} \boldsymbol{\beta}_{1}}$, siendo esta última la matriz inversa de la primera; es decir, ambas matrices definen codificaciones y decodificaciones directas, no desde la sombra sino sobre ella. Algo similar se podría decir de las matrices $[Q]_{\boldsymbol{\beta}^{\prime} \boldsymbol{\beta}_{1}^{\prime}}$ y $[Q]_{\boldsymbol{\beta}_{1}^{\prime} \boldsymbol{\beta}^{\prime}}$ en el contradominio, pero en relación con $[\boldsymbol{y}]_{\boldsymbol{\beta}^{\prime}}$ y $[\boldsymbol{y}]_{\boldsymbol{\beta}_{1}^{\prime}}$.

La Ecuación (2) parece sumamente simple, pero en casos más complejos considera composición de transformaciones con sus correspondientes codificaciones y decodificaciones, tal como lo ilustra Figura 1.

Pero, ¿cómo se calculan $[Q]_{\boldsymbol{\beta}^{\prime} \boldsymbol{\beta}_{1}^{\prime}}$ y $[P]_{\boldsymbol{\beta}_{1} \boldsymbol{\beta}}$ ? La respuesta a una de las matrices responde de manera similar a la otra, por lo que solamente se considerará la matriz $[P]_{\beta_{1} \beta}$. De acuerdo con la Ecuación (1), esta matriz codifica la información con respecto a la base $\beta_{1}$ en información con respecto a la base $\beta$.

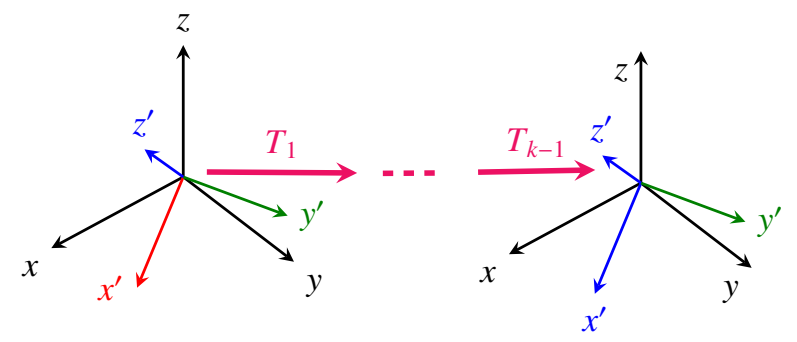

Figura 1: Secuencia de transformaciones aplicadas a diferentes espacios con diferentes codificaciones y decodificaciones, iniciando con la transformación $T_{1}$ y terminando con la transformación $T_{k-1}$, para obtener la transformación

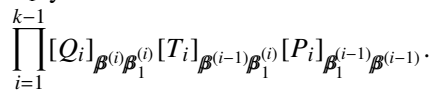

Si $[\boldsymbol{x}]_{\boldsymbol{\beta}_{1}}$ es la matriz de coordenadas del vector $i$-ésimo $\boldsymbol{\beta}_{i}^{(1)}$ en la base $\beta_{1}$, entonces

$$
[\boldsymbol{x}]_{\beta_{1}}=\left[\begin{array}{c}
0 \\
\vdots \\
0 \\
1 \\
0 \\
\vdots \\
0
\end{array}\right]_{\beta_{1}} \leftarrow i \text { - ésima componente, }
$$

y el producto $[P]_{\beta_{1} \beta}[x]_{\beta_{1}}$ da como resultado una matriz de coordenadas en la base $\boldsymbol{\beta}$, cuyas componentes son dadas por la columna $i$ de la matriz $[P]_{\boldsymbol{\beta}_{1} \boldsymbol{\beta}}$. Esto significa que $\boldsymbol{\beta}_{i}^{(1)}$ es combinación lineal de los elementos de la base $\boldsymbol{\beta} \mathrm{y}$, por consiguiente, en la sombra se tiene que

$$
\boldsymbol{\beta}_{i}^{(1)}=\sum_{k=1}^{n} p_{k i} \boldsymbol{\beta}_{k}, \forall i=1,2, \ldots, n
$$


donde, no debe olvidarse, en la sombra los vectores se expresan en base canónica. Puesto que ahora todo es en la sombra, entonces multiplicando en producto punto ambos lados de la Ecuación (3) por $\boldsymbol{\beta}_{j}$ se obtiene lo siguiente,

$$
\boldsymbol{\beta}_{i}^{(1)} \cdot \boldsymbol{\beta}_{j}=\sum_{k=1}^{n}\left(\boldsymbol{\beta}_{k} \cdot \boldsymbol{\beta}_{j}\right) p_{k i}, \forall i=1,2, \ldots, n
$$

Para una $i$ dada, este es un sistema de $n$ ecuaciones lineales con $n$ incógnitas que tiene solución única no trivial, pues se asume que los vectores en la base $\boldsymbol{\beta}$ son linealmente independientes. Considérese el caso relativamente simple en el que los vectores del conjunto generador $\beta$ son ortogonales, entonces la Ecuación (4) llega a ser $p_{j i}\left\|\boldsymbol{\beta}_{j}\right\|^{2}=\boldsymbol{\beta}_{i}^{(1)} \cdot \boldsymbol{\beta}_{j}$. Si existe ortonormalidad, entonces $p_{i j}=\boldsymbol{\beta}_{i} \cdot \boldsymbol{\beta}_{j}^{(1)}, \forall i, j=1,2, \ldots, n$.

Finalmente, el caso no trivial más simple se presenta cuando la transformación $T$ es la identidad, lo que significa que el dominio y codominio de ella son definidos por el mismo espacio de estímulos, y la matriz $[T]_{\boldsymbol{\beta}_{c} \boldsymbol{\beta}_{c}^{\prime}}$ de la transformación es la identidad con orden igual a la dimensión del espacio. Si además se asume que $\boldsymbol{\beta}_{1}$ y $\boldsymbol{\beta}_{1}^{\prime}$ representan a la misma base nueva, entonces de acuerdo con la Ecuación (2),

$$
[T]_{\boldsymbol{\beta}_{1} \boldsymbol{\beta}_{1}}=[Q]_{\boldsymbol{\beta}_{c} \boldsymbol{\beta}_{1}}[T]_{\boldsymbol{\beta}_{c} \boldsymbol{\beta}_{c}}[P]_{\boldsymbol{\beta}_{1} \boldsymbol{\beta}_{c}}
$$

lo que significa que la matriz de la transformación en la base $\boldsymbol{\beta}_{1}$ del dominio y contradominio es también la identidad, ya que $[Q]_{\boldsymbol{\beta}_{c} \boldsymbol{\beta}_{1}}[P]_{\boldsymbol{\beta}_{1} \boldsymbol{\beta}_{c}}$ es la matriz identidad, puesto que la matriz $[Q]_{\boldsymbol{\beta}_{c} \boldsymbol{\beta}_{1}}$ decodifica lo que la matriz $[P]_{\boldsymbol{\beta}_{1} \boldsymbol{\beta}_{c}}$ codifica. Ambas matrices rotan los puntos.

\subsection{Sistema Local de Coordenadas}

Debe decirse que no toda transformación con estructura matricial $[T]_{\beta \beta^{\prime}}[\boldsymbol{x}]_{\boldsymbol{\beta}}$ es necesariamente lineal. El ejemplo clásico es dado por la transformación afín $T: \mathbb{R}^{n} \rightarrow \mathbb{R}^{n}$ definida como $T(\boldsymbol{x})=\boldsymbol{x} R^{T}+\boldsymbol{c}, \boldsymbol{x}, \boldsymbol{c} \in \mathbb{R}^{n}$, siendo $\boldsymbol{c}$ un vector constante. Esta transformación se puede escribir en forma matricial usando el concepto de coordenadas homogeneas.

Cambiando la dimensión del dominio y codominio de la transformación hacia $\mathbb{R}^{n+1}$, de tal forma que $T_{h}: \mathbb{R}^{n+1} \rightarrow \mathbb{R}^{n+1}$ tenga la estructura $T_{h}(\boldsymbol{x}, 1)=(\boldsymbol{x}, 1) A^{T}, \boldsymbol{x} \in \mathbb{R}^{n}$, donde $A$ es una matriz de orden $n+1$, tal que

$$
A=\left[\begin{array}{cc}
R & c^{T} \\
\mathbf{0} & 1
\end{array}\right], \mathbf{0} \in \mathbb{R}^{n}, \mathbf{0}=(0,0, \ldots, 0)
$$

La imagen resultante después de aplicar la transformación al estímulo $\boldsymbol{x}$ resulta ser el vector

$$
\left(R_{11} x_{1}+\cdots+R_{1 n} x_{n}+c_{1}, \ldots, R_{n 1} x_{1}+\cdots+R_{n n} x_{n}+c_{n}, 1\right)
$$

que, después de eliminar la componente $n$-ésima, se transforma en el vector

$\left(R_{11} x_{1}+\cdots+R_{1 n} x_{n}+c_{1}, \ldots, R_{n 1} x_{1}+\cdots+R_{n n} x_{n}+c_{n}\right)=x R^{T}+c$

que es la imagen del vector $\boldsymbol{x}$ bajo la transformación no lineal original $T$, suponiendo que la fila $i$ de $R$ es dada por $R_{i}=$ $\left(R_{i 1}, \ldots, R_{\text {in }}\right), \forall i=1,2, \ldots, n$.
Al igual que en el caso de las transformaciones lineales, aunque en una forma más compleja, aquí se tiene una primera etapa de preprocesamiento de información, una segunda de procesamiento de ésta, y una última de posprocesamiento de ella. El efecto del vector $c$ consiste en trasladar el resultado obtenido por el término $x R^{T}$ en la transformación; es decir, moverlo hacia otro punto del dominio de $T$.

Por lo tanto, además de doblar el espacio, rotarlo, estirarlo, contraerlo, reflejarlo, etc., las transformaciones también son capaces de trasladar puntos en él. Por consiguiente, los sistemas de coordenadas no necesariamente están fijos con origen común al del Sistema Universal de Coordenadas.

Se pueden definir sistemas de coordenadas con origen en un punto $\boldsymbol{c}$, diferente del vector nulo, referido al Sistema Universal de Coordenadas. Es más, estos sistemas de coordenadas pueden incluso estar en movimiento con aceleración no necesariamente constante; es decir, sujetos a fuerzas físicas (¿psicológicas?). Estos sistemas de coordenadas cuentan con su propia base, por supuesto siempre referidas a la sombra canónica del Sistema Universal de Coordenadas, o aún a su propia sombra canónica local, y se conocen como Sistemas de Coordenadas Locales.

Es posible descomponer la matriz $A$ en dos matrices, una de rotación $R_{\alpha}$ y otra de traslación $T_{c}$, la primera de las cuales rota un punto del espacio alrededor de un eje con ángulos $\boldsymbol{\alpha}$ referidos al Sistema Universal de Coordenadas, y la segunda de las cuales produce un desplazamiento $c$ a cualquier punto del espacio, otra vez bajo la sombra del Sistema de Coordenadas Universal. Es decir,

$$
R_{\alpha}=\left[\begin{array}{cc}
R & \mathbf{0}^{T} \\
\mathbf{0} & 1
\end{array}\right], T_{c}=\left[\begin{array}{cc}
I & c^{T} \\
\mathbf{0} & 1
\end{array}\right]
$$

Claramente, $(x, 1) R_{\alpha}^{T}=\left(x R_{\alpha}^{T}, 1\right)$ y $(x, 1) T_{c}^{T}=(x+c, 1)$. Sin embargo, $(x, 1) R_{\alpha}^{T} T_{c}^{T}=\left(x R_{\alpha}^{T}+c, 1\right)$ es completamente diferente de $(x, 1) T_{c}^{T} R_{\alpha}^{T}=(x+c, 1) R_{\alpha}^{T}=\left((x+c) R_{\alpha}^{T}, 1\right)$. Es decir, el producto de la matrices $R_{\alpha}$ y $T_{c}$ no es conmutativo, excepto para ciertas rotaciones, como se escribirá a continuación.

Supóngase que, en el Sistema Universal de Coordenadas, los puntos en el espacio rotan alrededor de un eje que pasa por el origen del sistema. Sea $\boldsymbol{v}$ el vector de dirección de este eje. La rotación alrededor de este eje se denotará como $R_{v} \mathrm{y}$, dado $x$, la rotación de este punto alrededor del eje propuesto es $(x, 1) R_{v}^{T}$.

Obsérvese que, puesto que el eje de rotación pasa por el origen del Sistema Universal de Coordenadas, esta rotación asume un sistema de coordenadas nuevo cuyo origen coincide con el del Sistema Universal de Coordenadas. Es más, para mayor simplicidad, podría suponerse que el eje de rotación define uno de los elementos de la base del nuevo sistema.

Si el punto rotado en la forma que se describe en el párrafo anterior se traslada ahora al punto $c$, referido al Sistema de Coordenadas Universal, se obtiene el punto rotado y trasladado $\left(x R_{v}^{T}, 1\right) T_{c}^{T}=\left(x R_{v}^{T}+c, 1\right)$.

$\mathrm{Si}$ ahora, en lugar de rotar el punto $\boldsymbol{x}$ alrededor del eje definido, se desplaza primeramente el punto usando el vector $c$ y, puesto que hay que rotar este punto nuevo alrededor del eje definido por $\boldsymbol{v} \sin$ afectar el resultado anterior, entonces el eje de rotación es también trasladado usando el vector $\boldsymbol{c}$, por lo que 
el nuevo eje de rotación pasa por $c$ y tiene vector de dirección $v$.

El punto a rotar alrededor del eje que pasa por $c$ con vector de dirección $\boldsymbol{v}$, referido al Sistema de Coordenadas Universal, es ahora $\boldsymbol{x}+\boldsymbol{c}$. Ya que la rotación es local, alrededor del eje que pasa por $\boldsymbol{c}$, con vector de dirección $\boldsymbol{v}$, entonces la rotación del punto $c$ sigue siendo el mismo punto; es decir, $(\boldsymbol{c}, 1) R_{v, l o c a l}^{T}=$ $(c, 1)$.

Sin pérdida de generalidad, se escoge el punto $c$ como el origen del sistema de coordenadas local, por lo que referido a este sistema el punto $\boldsymbol{x}+\boldsymbol{c}$ tiene coordenadas iguales a las de $\boldsymbol{x}$, visto este último punto desde el Sistema de Coordenadas Universal.

Así que rotar $\boldsymbol{x}+\boldsymbol{c}$ alrededor del eje que pasa por $\boldsymbol{c}$ y tiene vector de dirección $\boldsymbol{v}$ es lo mismo que rotar el vector $\boldsymbol{x}$ visto desde el sistema de coordenadas local, asumiendo que éste tiene ejes paralelos a los del Sistema de Coordenadas Universal, y esta rotación es $(\boldsymbol{x}, 1) R_{\boldsymbol{v} \text {, local }}=\left(\boldsymbol{x} R_{\text {local }}^{T}, 1\right)$. Finalmente, el punto trasladado y rotado desde el nuevo sistema es $(\boldsymbol{x}+\boldsymbol{c}, 1) R_{v, \text { local }}^{T}=$ $\left((x+c) R_{\text {local }}^{T}, 1\right)=\left(x R_{\text {local }}^{T}+c, 1\right)$. Debido a que $R_{\text {local }}^{T}$ es idéntica a la rotación $R_{v}^{T}$, se tiene entonces que el orden en que se realizan las operaciones no importa.

\section{Ecuaciones básicas de la dinámica del sistema de drones más carga}

En el sistema definido por los drones, más la carga a transportar por ellos, existe por supuesto un Sistema de Coordenadas Universal fijo, un Sistema de Coordenadas Local ubicado en el centro de masa de la carga que se mueve, además de tantos Sistemas de Coordenadas Locales en movimiento como drones se consideren en la dinámica del sistema completo.

Supóngase que se tienen $n$ drones, siendo $m_{i}$ la masa del dron $i$-ésimo y que, con respecto al Sistema de Coordenadas Universal, la posición del centro de masa de la carga es dada por el vector $\boldsymbol{x}_{0}$, o bien por la matriz de coordenadas en base canónica denotada por $\left[\boldsymbol{x}_{0}\right]_{\boldsymbol{\beta}_{c}}$. Además, el centro de masa del dron $i$ tiene position $\boldsymbol{x}_{0_{i}}$ con respecto al Sistema de Coordenadas Universal, o bien es dado por la matriz de coordenadas en base canónica, representada por $\left[\boldsymbol{x}_{0_{i}}\right]_{\boldsymbol{\beta}_{c}}$, lo que Figura 2 ilustra.

Los sistemas locales definidos por el centro de masa del objeto a levantar y por el centro de masa de cada uno de los drones involucrados pueden rotar, e incluso ser trasladados, no siendo este el caso para el Sistema de Coordenadas Universal, el cual siempre conserva la base canónica $\boldsymbol{\beta}_{c}$, y no precisamente bajo la sombra.

Esto significa que además de las bases canónicas $\boldsymbol{\beta}_{c_{c m}}$ y $\boldsymbol{\beta}_{c_{d_{i}}}$ en los sistemas locales del centro de masa de la carga y del dron $i$-ésimo, respectivamente, existen bases locales rotadas y trasladadas que deben monitorearse continuamente en la descripción de la dinámica del sistema en su conjunto, o en partes de él.

Sean $A_{0}$ y $A_{i}$, respectivamente, las matrices que contienen traslación y rotación para los sistemas locales de coordenadas del centro de masa y del dron $i$-ésimo. De acuerdo con la definición de la matriz para una transformación afín dada por

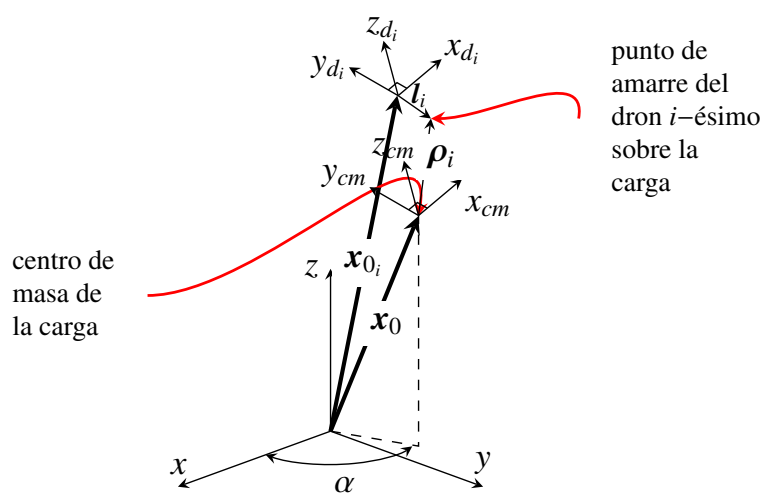

Figura 2: Sistemas de Coordenadas Universal (base canónica $\boldsymbol{\beta}_{c}$ ) y Locales correspondientes al centro de masa de la carga (base canónica $\boldsymbol{\beta}_{c_{c m}}$ ) y al centro de masa del $i$-ésimo dron (base canónica $\boldsymbol{\beta}_{c_{d_{d}}}$ ). El ángulo $\alpha$ es una de las diferentes variables angulares usadas para indicar dirección.

la Ecuación (5), se tiene entonces que

$$
A_{0}=\left[\begin{array}{cc}
{\left[R_{0}\right]_{\boldsymbol{\beta}_{c_{c m}} \boldsymbol{\beta}_{r_{c m}}}} & {\left[\boldsymbol{x}_{0}\right]_{\boldsymbol{\beta}_{c}}} \\
{[\mathbf{0}]_{\boldsymbol{\beta}_{c c m}}^{T}} & 1
\end{array}\right] ; A_{i}=\left[\begin{array}{cc}
{\left[R_{i}\right]_{\boldsymbol{\beta}_{c_{d_{i}}} \boldsymbol{\beta}_{r_{d_{i}}}}} & {\left[\boldsymbol{x}_{0_{i}}\right]_{\boldsymbol{\beta}_{c}}} \\
{[\mathbf{0}]_{\boldsymbol{\beta}_{c_{d_{i}}}^{T}}^{T}} & 1
\end{array}\right]
$$

Nótese que $A_{0}$ y $A_{i}$ operan sobre información local, proveniente de los sistemas de coordenadas locales de los centros de masa de la carga y del dron $i$-ésimo, respectivamente. En otras palabras, $A_{0}$ acepta información de la forma $[\boldsymbol{x}]_{\boldsymbol{\beta}_{c c m}}$, cuando un objeto visto desde el Sistema Universal de Coordenadas tiene posición $[\boldsymbol{x}]_{\boldsymbol{\beta}_{c}}$. Por otro lado, $A_{i}$ recibe estímulos $[\boldsymbol{x}]_{\boldsymbol{\beta}_{c_{d_{i}}}}$, cuando un objeto visto desde el Sistema de Coordenadas Universal tiene posición $[\boldsymbol{x}]_{\boldsymbol{\beta}_{c}}$.

Sin embargo, las respuestas de ambas matrices pertenecen al mismo espacio; a saber, el definido por el Sistema de Coordenadas Universal. Así,

$$
A_{0}\left[\begin{array}{c}
{[\boldsymbol{x}]_{\boldsymbol{\beta}_{c_{c m}}}} \\
1
\end{array}\right]=\left[\begin{array}{c}
{\left[\boldsymbol{R}_{0}\right]_{\boldsymbol{\beta}_{c_{c m}} \boldsymbol{\beta}_{r_{c m}}}[\boldsymbol{x}]_{\boldsymbol{\beta}_{c_{c m}}}+\left[\boldsymbol{x}_{0}\right]_{\boldsymbol{\beta}_{c}}} \\
1
\end{array}\right]
$$

proporciona información acerca de la posición de $[\boldsymbol{x}]_{\boldsymbol{\beta}_{c_{c m}}}$, después de ser rotado en el sistema local y trasladado (o viceversa), visto desde el Sistema Universal de Coordenadas con base canónica. Desde la sombra, se hace la suma referida a la base canónica del Sistema Universal de Coordenadas. Similarmente,

$$
A_{i}\left[\begin{array}{c}
{[\boldsymbol{x}]_{\boldsymbol{\beta}_{c_{d_{i}}}}} \\
1
\end{array}\right]=\left[\begin{array}{c}
{\left[R_{i}\right]_{\boldsymbol{\beta}_{c_{d_{i}}} \boldsymbol{\beta}_{r_{d_{i}}}}[\boldsymbol{x}]_{\boldsymbol{\beta}_{c_{d_{i}}}}+\left[\boldsymbol{x}_{0_{i}}\right]_{\boldsymbol{\beta}_{c}}} \\
1
\end{array}\right]
$$

Específicamente, el punto de amarre $i$ visto desde el sistema local, ubicado éste en el centro de masa de la carga, está sobre la carga y su posición es dada por $\left[\boldsymbol{\rho}_{i}\right]_{\boldsymbol{\beta}_{c c m}}$. Sin embargo, como lo ilustra Figura 2, este mismo punto tiene posición $\left[\boldsymbol{l}_{i}\right]_{\boldsymbol{\beta}_{c_{d_{i}}}}$ cuando se ve desde el sistema local definido por el dron $i$. Por lo tanto, $[\boldsymbol{x}]_{\boldsymbol{\beta}_{\boldsymbol{\beta}_{c m}}}=\left[\boldsymbol{\rho}_{i}\right]_{\boldsymbol{\beta}_{c c m}}$ y $[\boldsymbol{x}]_{\boldsymbol{\beta}_{c_{d_{i}}}}=\left[\boldsymbol{l}_{i}\right]_{\boldsymbol{\beta}_{c_{d_{i}}}}$ en las correspondientes ecuaciones matriciales anteriores. 
Recordando que aparecen operaciones desde la sombra del Sistema Universal de Coordenadas, se tienen las siguientes ecuaciones sumamente elementales, pero no por ello sin importancia,

$$
\begin{aligned}
& {\left[\boldsymbol{x}_{a_{i}}\right]_{\boldsymbol{\beta}_{c}}=\left[\boldsymbol{x}_{0}\right]_{\boldsymbol{\beta}_{c}}+\left[\boldsymbol{\rho}_{i}\right]_{\boldsymbol{\beta}_{c c m}}} \\
& {\left[\boldsymbol{l}_{i}\right]_{\boldsymbol{\beta}_{c_{d_{i}}}}=\left[\boldsymbol{x}_{a_{i}}\right]_{\boldsymbol{\beta}_{c}}-\left[\boldsymbol{x}_{0_{i}}\right]_{\boldsymbol{\beta}_{c}}}
\end{aligned}
$$

y tras algunas operaciones algebraicas, después de sustituir la expresión para $\left[\boldsymbol{x}_{a_{i}}\right]_{\boldsymbol{\beta}_{c}}$ de la primera ecuación en la segunda ecuación, se obtiene

$$
\left[\boldsymbol{\rho}_{i}\right]_{\boldsymbol{\beta}_{c c m}}-\left[\boldsymbol{l}_{i}\right]_{\boldsymbol{\beta}_{c_{d_{i}}}}=\left[\boldsymbol{x}_{0_{i}}\right]_{\boldsymbol{\beta}_{c}}-\left[\boldsymbol{x}_{0}\right]_{\boldsymbol{\beta}_{c}}
$$

por lo que la posición del dron $i$-ésimo es simplemente,

$$
\left[\boldsymbol{x}_{0_{i}}\right]_{\boldsymbol{\beta}_{c}}=\left[\boldsymbol{x}_{0}\right]_{\boldsymbol{\beta}_{c}}+\left[\boldsymbol{\rho}_{i}\right]_{\boldsymbol{\beta}_{c c m}}-\left[\boldsymbol{l}_{i}\right]_{\boldsymbol{\beta}_{c_{d_{i}}}}
$$

¡Ya está...! la posición del dron $i$-ésimo está determinada por las posiciones del centro de masa de la carga referida al Sistema Universal de Coordenadas, y la posición del punto de amarre $i$ según se observa desde los sistemas locales de los centros de masa de la carga y del dron $i$-ésimo (siempre bajo la sombra del Sistema de Coordenadas Universal). Si existen rotaciones en los sistemas locales, entonces en las ecuaciones anteriores las posiciones locales deben incluir el producto matricial rotacional de las matrices $A_{0}$ y $A_{i}$ solamente, ya que las traslaciones están incluidas en $\left[\boldsymbol{x}_{0}\right]_{\boldsymbol{\beta}_{c}}$ y $\left[\boldsymbol{x}_{0_{i}}\right]_{\boldsymbol{\beta}_{c}}$. Así,

$$
\left[\boldsymbol{x}_{0_{i}}\right]_{\boldsymbol{\beta}_{c}}=\left[\boldsymbol{x}_{0}\right]_{\boldsymbol{\beta}_{c}}+\left[R_{0}\right]_{\boldsymbol{\beta}_{c c m} \boldsymbol{\beta}_{r_{c m}}}\left[\boldsymbol{\rho}_{i}\right]_{\boldsymbol{\beta}_{c_{c m}}}-\left[R_{i}\right]_{\boldsymbol{\beta}_{c_{d_{i}}} \boldsymbol{\beta}_{d_{i}}}\left[\boldsymbol{l}_{i}\right]_{\boldsymbol{\beta}_{c_{d_{i}}}}
$$

\section{Formalismo Lagrangiano}

Como seguramente se habrá adivinado, el movimiento del sistema involucra traslaciones y rotaciones. Por lo que se intuye que habrá energías cinéticas relacionadas con estos tipos de movimiento. De hecho, el formalismo Lagrangiano requiere de la definición de la energía cinética y de la energía potencial del sistema, para construir lo que se conoce como Lagrangiana. ¿Cómo especificarlas en el caso que aquí se analiza? La Ecuación (7) da la respuesta, como se escribirá a continuación.

\subsection{Movimiento rotacional}

La energía cinética rotacional de un cuerpo rígido se puede obtener a través de la definición misma, que implica la integración de energías cinéticas como sigue,

$$
d K_{r}=\frac{1}{2} \rho(x, y, z) v^{2} d x d y d z
$$

donde $\rho(x, y, z)$ define la densidad del cuerpo (masa por unidad de volumen) y $v$ representa la velocidad lineal del elemento de masa diferencial $d m=\rho(x, y, z) d x d y d z$. La integral se toma sobre la geometría completa del cuerpo rígido, por lo que

$$
\begin{aligned}
K_{r} & =\frac{1}{2} \int_{V} \rho \boldsymbol{v} \cdot \boldsymbol{v} d x d y d z \\
& =\frac{1}{2} \int_{V} \rho d V(\boldsymbol{\omega} \times \boldsymbol{r}) \cdot(\boldsymbol{\omega} \times \boldsymbol{r})
\end{aligned}
$$

Sin embargo, el triple producto escalar (triple debido a que existen tres operandos) es invariante bajo el desplazamiento circular de los tres operandos, de tal manera que

$$
\begin{aligned}
(\omega \times \boldsymbol{r}) \cdot(\omega \times \boldsymbol{r}) & =\boldsymbol{a} \cdot(\boldsymbol{\omega} \times \boldsymbol{r}), \text { donde } \boldsymbol{a}=\omega \times \boldsymbol{r} \\
& =\omega \cdot(\boldsymbol{r} \times(\omega \times \boldsymbol{r})) \\
& =\omega \cdot\left(r^{2} \omega-(\boldsymbol{r} \cdot \omega) \boldsymbol{r}\right)
\end{aligned}
$$

y la igualdad $\boldsymbol{a} \times(\boldsymbol{b} \times \boldsymbol{c})=\boldsymbol{b}(\boldsymbol{a} \cdot \boldsymbol{c})-\boldsymbol{c}(\boldsymbol{a} \cdot \boldsymbol{b})$ ha sido usada $(\boldsymbol{a}=\boldsymbol{c}=\boldsymbol{r}$ y $\boldsymbol{b}=\omega)$.

Para el movimiento de un cuerpo rígido, la velocidad angular $\omega$ no depende de la posición, sino que depende solamente del tiempo $t$, por lo que

$$
K_{r}=\frac{1}{2} \omega \cdot \int_{V} \rho d V\left(r^{2} \omega-(\boldsymbol{r} \cdot \omega) \boldsymbol{r}\right)
$$

El factor $r^{2} \omega-(\boldsymbol{r} \cdot \omega) \boldsymbol{r}$ dentro de la integral es un vector que puede descomponerse como sigue,

$$
\begin{aligned}
r^{2} \omega-(\boldsymbol{r} \cdot \omega) \boldsymbol{r}= & \left(r^{2} \omega_{x}, r^{2} \omega_{y}, r^{2} \omega_{z}\right)-((\boldsymbol{r} \cdot \boldsymbol{\omega}) x,(\boldsymbol{r} \cdot \boldsymbol{\omega}) y,(\boldsymbol{r} \cdot \boldsymbol{\omega}) z) \\
= & \left(\left(y^{2}+z^{2}\right) \omega_{x}-x y \omega_{y}-x z \omega_{z},\left(x^{2}+z^{2}\right) \omega_{y}-x y \omega_{x}\right. \\
& \left.-y z \omega_{z},\left(x^{2}+y^{2}\right) \omega_{z}-x z \omega_{x}-y z \omega_{y}\right) \\
= & {\left[\begin{array}{ccc}
y^{2}+z^{2} & -x y & -x z \\
-x y & x^{2}+z^{2} & -y z \\
-x z & -y z & x^{2}+y^{2}
\end{array}\right]\left[\begin{array}{c}
\omega_{x} \\
\omega_{y} \\
\omega_{z}
\end{array}\right] } \\
= & \mathbb{M} \omega^{T}
\end{aligned}
$$

donde la matriz $\mathbb{M}$ se conoce como tensor de inercia. Así que,

$$
\begin{aligned}
& K_{r}=\frac{1}{2} \omega\left(\int_{V} \rho \mathbb{M} d V\right) \omega^{T} \\
& =\frac{1}{2} \omega \| \omega^{T}
\end{aligned}
$$

donde $\mathbb{I}=\int_{V} \rho \mathbb{M} d V$.

\subsection{Energía cinética total del sistema drones más carga}

A continuación, la energía cinética total del sistema se compone de energías cinéticas de traslación y de rotación de la carga y de los drones; es decir,

$$
\begin{aligned}
& K=\frac{1}{2} M\left\|\left[\dot{x_{0}}\right]_{\boldsymbol{\beta}_{c}}\right\|^{2}+\frac{1}{2}[\omega]_{\boldsymbol{\beta}_{c c m}}^{T} \mathbb{I}_{0}[\omega]_{\boldsymbol{\beta}_{c c m}}+\frac{1}{2} \sum_{i=1}^{n} m_{i}\left\|\left[\dot{x_{0 i}}\right]_{\boldsymbol{\beta}_{c}}\right\|^{2}+ \\
& \frac{1}{2} \sum_{i=1}^{n}\left[\omega_{i}\right]_{\boldsymbol{\beta}_{d_{i}}}^{T} \mathbb{I}_{i}\left[\omega_{i}\right]_{\boldsymbol{\beta}_{c_{d_{i}}}} \\
& =\frac{1}{2} M\left\|\left[\dot{x_{0}}\right]_{\boldsymbol{\beta}_{c}}\right\|^{2}+\frac{1}{2}[\omega]_{\boldsymbol{\beta}_{c c m}}^{T} \mathbb{I}_{0}[\omega]_{\boldsymbol{\beta}_{c c m}}+ \\
& \frac{1}{2} \sum_{i=1}^{n} m_{i}\left\|\left[\dot{\boldsymbol{x}_{0}}\right]_{\boldsymbol{\beta}_{c}}+\frac{.}{\left[R_{0}\right]_{\boldsymbol{\beta}_{c c m} \boldsymbol{\beta}_{r c m}}\left[\boldsymbol{\rho}_{i}\right]_{\boldsymbol{\beta}_{c c m}}}-\overline{\left[R_{i}\right]_{\boldsymbol{\beta}_{d_{i}} \boldsymbol{\beta}_{d_{d_{i}}}\left[\boldsymbol{l}_{i}\right]}}\right\|_{\boldsymbol{\beta}_{c_{i}}} \|^{2}+ \\
& \frac{1}{2} \sum_{i=1}^{n}\left[\omega_{i}\right]_{\boldsymbol{\beta}_{c_{i}}}^{T} \mathbb{I}_{i}\left[\omega_{i}\right]_{\boldsymbol{\beta}_{d_{d_{i}}}}
\end{aligned}
$$

donde se hace uso de la notación $\frac{d}{d t}[\cdot]=[\cdot]$. ¡Dios, qué engorrosa ecuación para determinar la energía cinética total! ¿No habrá forma de simplificarla aunque sea un poquito? Veamos. Los términos $\left[R_{0}\right]_{\boldsymbol{\beta}_{c c m} \boldsymbol{\beta}_{r c m}}\left[\boldsymbol{\rho}_{i}\right]_{\boldsymbol{\beta}_{c_{c m}}}$ y $\left[R_{i}\right]_{\boldsymbol{\beta}_{c_{d_{i}}} \boldsymbol{\beta}_{r_{d_{i}}}}\left[\boldsymbol{l}_{i}\right]_{\boldsymbol{\beta}_{c_{d_{i}}}}$ llaman un 
poco la atención, y básicamente se refieren a la forma en que se ven los vectores $\left[\boldsymbol{\rho}_{i}\right]_{\boldsymbol{\beta}_{c c m}}$ y $\left[\boldsymbol{l}_{i}\right]_{\boldsymbol{\beta}_{d_{d_{i}}}}$ en bases canónicas de las coordenadas locales del centro de masa de la carga y del dron $i$, respectivamente, desde el punto de vista de las bases de los sistemas de coordenadas rotados, $\boldsymbol{\beta}_{r_{c m}}$ y $\boldsymbol{\beta}_{r_{d_{i}}}$.

Cada uno de estos vectores define una variable nueva desde el enfoque de los sistemas rotados; a saber, $\left[\boldsymbol{\rho}_{i}\right]_{\boldsymbol{\beta}_{r_{c m}}}$ y $\left[\boldsymbol{l}_{i}\right]_{\boldsymbol{\beta}_{r_{d}}}$, respectivamente. Variables observadas desde el correspondiente sistema de coordenadas rotado ¡Sin matrices de rotación presentes! El planteamiento es muy similar al fenómeno de un cuerpo rígido girando alrededor de un eje sobre él mismo, el cual define uno de los ejes del sistema de coordenadas local, y que además se mueve a una cierta velocidad con respecto a un Sistema de Coordenadas Universal fijo.

No es difícil darse cuenta que si $\boldsymbol{r}_{0}$ es la posición del origen del sistema de coordenadas local (localizado en el cuerpo rígido mismo), visto desde el Sistema de Coordenadas Universal, $\boldsymbol{r}^{\prime}$ es la posición de un punto sobre el cuerpo rígido, visto desde el sistema de coordenadas local, y que $\boldsymbol{r}$ es la posición de este mismo punto, según el Sistema de Coordenadas Universal, entonces $\boldsymbol{r}=\boldsymbol{r}_{0}+\boldsymbol{r}^{\prime}$, por lo que la velocidad del punto sobre el cuerpo rígido, según el Sistema de Coordenadas Universal, es $\boldsymbol{v}=\boldsymbol{v}_{0}+\boldsymbol{v}^{\prime}$, pero como el punto sobre el cuerpo rígido rota a una velocidad angular $\omega$, desde el punto de vista del sistema de coordenadas local, entonces $\boldsymbol{v}=\boldsymbol{v}_{0}+\boldsymbol{\omega} \times \boldsymbol{r}^{\prime}$. Si el cuerpo rígido no se traslada con respecto al Sistema Universal de Coordenadas, lo que podría equivaler también a que el Sistema Universal de Coordenadas se ubica sobre el cuerpo rígido, entonces $\boldsymbol{v}=\boldsymbol{\omega} \times \boldsymbol{r}^{\prime}$, y la descripción del movimiento es mucho más simple. Así, volviendo al tema de interés, Ecuación (9) se reescribe como sigue,

$$
\begin{aligned}
K= & \frac{1}{2} M\left\|\left[\dot{x_{0}}\right]_{\boldsymbol{\beta}_{c}}\right\|^{2}+\frac{1}{2}[\omega]_{\boldsymbol{\beta}_{c_{c} m}}^{T} \mathbb{I}_{0}[\omega]_{\boldsymbol{\beta}_{c c m}}+ \\
& \frac{1}{2} \sum_{i=1}^{n} m_{i}\left\|\left[\dot{\boldsymbol{x}_{0}}\right]_{\boldsymbol{\beta}_{c}}+\left[\dot{\boldsymbol{\rho}_{i}}\right]_{\boldsymbol{\beta}_{r_{c m}}}-\left[\dot{\boldsymbol{l}}_{i}\right]_{\boldsymbol{\beta}_{r_{d_{i}}}}\right\|^{2}+\frac{1}{2} \sum_{i=1}^{n}\left[\omega_{i}\right]_{\boldsymbol{\beta}_{c_{d_{i}}}^{T}}^{T} \mathbb{I}_{i}\left[\omega_{i}\right]_{\boldsymbol{\beta}_{c_{d_{i}}}}
\end{aligned}
$$

\subsection{Energía potencial total del sistema drones, cuerdas, carga}

Por otro lado, las Ecuaciones (4.3) proporcionan la energía potencial de la carga y del dron $i$, respectivamente

$$
U_{0}=M_{0} g\left[\boldsymbol{\beta}_{3}\right]_{\boldsymbol{\beta}_{c}}^{T}\left[\boldsymbol{x}_{0}\right]_{\boldsymbol{\beta}_{c}} ; U_{i}=m_{i} g\left[\boldsymbol{\beta}_{3}\right]_{\boldsymbol{\beta}_{c}}^{T}\left[\boldsymbol{x}_{0_{i}}\right]_{\boldsymbol{\beta}_{c}}
$$

por lo que Ecuación (11) da la energía potencial total del sistema,

$$
U=M_{0} g\left[\boldsymbol{\beta}_{3}\right]_{\boldsymbol{\beta}_{c}}^{T}\left[\boldsymbol{x}_{0}\right]_{\boldsymbol{\beta}_{c}}+\sum_{i=1}^{n} m_{i} g\left[\boldsymbol{\beta}_{3}\right]_{\boldsymbol{\beta}_{c}}^{T}\left[\boldsymbol{x}_{0_{i}}\right]_{\boldsymbol{\beta}_{c}}
$$

\subsection{Lagrangiana del sistema drones, cuerdas más carga}

En conclusión, Ecuación (12) es la Lagrangiana del sistema completo de drones más carga,

$$
\begin{aligned}
\mathcal{L}= & K-U \\
= & \frac{1}{2} M_{0}\left\|\left[\dot{\boldsymbol{x}_{0}}\right]_{\boldsymbol{\beta}_{c}}\right\|^{2}+\frac{1}{2}[\boldsymbol{\omega}]_{\boldsymbol{\beta}_{c_{c m}}}^{T} \mathbb{I}_{0}[\boldsymbol{\omega}]_{\boldsymbol{\beta}_{c_{c m}}}+ \\
& \frac{1}{2} \sum_{i=1}^{n} m_{i}\left\|\left[\dot{\boldsymbol{x}}_{0_{i}}\right]_{\boldsymbol{\beta}_{c_{d_{i}}}}\right\|^{2}+\frac{1}{2} \sum_{i=1}^{n}\left[\boldsymbol{\omega}_{i}\right]_{\boldsymbol{\beta}_{c_{d_{i}}}^{T}} \mathbb{I}_{i}\left[\boldsymbol{\omega}_{i}\right]_{\boldsymbol{\beta}_{c_{d_{i}}}}- \\
& M_{0} g\left[\boldsymbol{\beta}_{3}\right]_{\boldsymbol{\beta}_{c}}^{T}\left[\boldsymbol{x}_{0}\right]_{\boldsymbol{\beta}_{c}}-\sum_{i=1}^{n} m_{i} g\left[\boldsymbol{\beta}_{3}\right]_{\boldsymbol{\beta}_{c}}^{T}\left[\boldsymbol{x}_{0_{i}}\right]_{\boldsymbol{\beta}_{c}}
\end{aligned}
$$

\subsection{Variables del sistema drones, cuerdas más carga}

Las variables del sistema drones, cuerdas más carga son los vectores de posición y sus correspondientes velocidades, $\left[\boldsymbol{x}_{0}\right]_{\boldsymbol{\beta}_{c}},\left[\dot{\boldsymbol{x}_{0}}\right]_{\boldsymbol{\beta}_{c}},\left[\boldsymbol{x}_{0_{i}}\right]_{\boldsymbol{\beta}_{c_{d_{i}}}},\left[\dot{\boldsymbol{x}_{0_{i}}}\right]_{\boldsymbol{\beta}_{c_{d_{i}}}},\left[\boldsymbol{l}_{i}\right]_{\boldsymbol{\beta}_{c_{d_{i}}}},\left[\dot{\boldsymbol{l}}_{i}\right]_{\boldsymbol{\beta}_{c_{d_{i}}}}$, además de las matrices de rotación $\left[R_{0}\right]_{\boldsymbol{\beta}_{c c m} \boldsymbol{\beta}_{r_{c m}}}$ y $\left[R_{i}\right]_{\boldsymbol{\beta}_{c_{d_{i}}} \boldsymbol{\beta}_{r_{d_{i}}}}$ que contienen a las posiciones angulares de las cuales se derivan las velocidades angulares $[\boldsymbol{\omega}]_{\boldsymbol{\beta}_{c c m}},\left[\boldsymbol{\omega}_{i}\right]_{\boldsymbol{\beta}_{c_{d_{i}}}}$, respectivamente .

Con ellas, es posible calcular el gradiente de la Lagrangiana, además de las variaciones temporales de las componentes de este gradiente, los cuales son de suma importancia para determinar las ecuaciones del movimiento del sistema completo, siguiendo el formalismo de Lagrange.

\subsection{Gradiente de Lagrangiana de sistema drones, cuerdas, carga}

Por cuestiones de espacio, en lo que sigue no se desglosará la forma en que se obtienen las componentes del gradiente de la Lagrangiana, ni la totalidad de las componentes, sino solamente algunas de ellas. Siguiendo el formalismo Lagrangiano, algunas de estas componentes del gradiente se derivan con respecto al tiempo para obtener finalmente las ecuaciones dinámicas. Así, tomando en cuenta Ecuación (12) se tiene

$$
\begin{aligned}
& \frac{\partial \mathcal{L}}{\partial\left[\dot{\boldsymbol{x}_{0}}\right]_{\boldsymbol{\beta}_{c}}}=\left(M_{0}+\sum_{i=1}^{n} m_{i}\right)\left[\dot{\boldsymbol{x}_{0}}\right]_{\boldsymbol{\beta}_{c}}+ \\
& \sum_{i=1}^{n} m_{i}\left(\left[\dot{\boldsymbol{\rho}}_{i}\right]_{\boldsymbol{\beta}_{r_{c m}}}-\left[\dot{\boldsymbol{i}}_{i}\right]_{\boldsymbol{\beta}_{r_{d_{i}}}}\right) \\
& \text { por Ecuación (7) } \\
& =M_{T}\left[\dot{x_{0}}\right]_{\boldsymbol{\beta}_{c}}+ \\
& M_{T}=M_{0}+\sum_{i=1}^{n} m_{i} \\
& \sum_{i=1}^{n} m_{i}\left(\left[\dot{\boldsymbol{\rho}}_{i}\right]_{\boldsymbol{\beta}_{r_{c m}}}-\left[\dot{\boldsymbol{l}}_{i}\right]_{\boldsymbol{\beta}_{r_{d_{i}}}}\right)
\end{aligned}
$$$$
\frac{\partial \mathcal{L}}{\partial\left[\dot{i}_{i}\right]_{\boldsymbol{\beta}_{r_{d_{i}}}}}=-m_{i}\left[\dot{\boldsymbol{x}_{0_{i}}}\right]_{\boldsymbol{\beta}_{c_{d_{i}}}}
$$

por Ecuación (7) y Ecuación (12)

y la variación de la Lagrangiana con respecto a un cambio en los ángulos de rotación de la carga se calcula como sigue, después de aplicar principios variacionales 


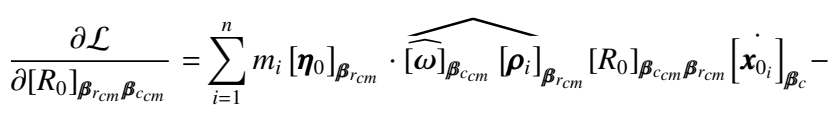

$$
\begin{aligned}
& \sum_{i=1}^{n} m_{i} g\left[\boldsymbol{\eta}_{0}\right]_{\boldsymbol{\beta}_{r c m}} \cdot{\widehat{\left[\boldsymbol{\rho}_{i}\right.}}_{\boldsymbol{\beta}_{r c m}}\left[R_{0}\right]_{\boldsymbol{\beta}_{c c m} \boldsymbol{\beta}_{r c m}}\left[\boldsymbol{\beta}_{3}\right]_{\boldsymbol{\beta}_{c}}
\end{aligned}
$$

donde $\left[\boldsymbol{\eta}_{0}\right]_{\boldsymbol{\beta}_{r_{c m}}}$ representa el desplazamiento en una trayectoria virtual según la teoría variacional. Se obtiene una ecuación similar para los ángulos de rotación de cada uno de los drones.

\section{Ecuaciones dinámicas del sistema drones, cuerdas, carga}

Se está ahora en posición de obtener las ecuaciones de movimiento del sistema que, de acuerdo con los cánones dictados por la mecánica clásica, son suficientes para describir la manera en que el sistema completo se mueve, siempre y cuando se proporcionen las condiciones iniciales de las diferentes variables, justo cuando el observador visualiza por primera vez el fenómeno analizado en este artículo. Así, se puede demostrar que una porción del sistema de ecuaciones diferenciales acopladas es la siguiente,

$$
\begin{aligned}
& M_{T}\left[\ddot{\boldsymbol{x}_{0}}\right]_{\boldsymbol{\beta}_{c}}+\sum_{i=1}^{n} m_{i}\left(-\widehat{\left[R_{0}\right]_{\boldsymbol{\beta}_{r c m} \boldsymbol{\beta}_{c c m}}\left[\boldsymbol{\rho}_{i}\right.}\right]_{\boldsymbol{\beta}_{r c m}}[\dot{\boldsymbol{\omega}}]_{\boldsymbol{\beta}_{c_{c m}}}- \\
& \left.\widehat{[\omega}_{\boldsymbol{\beta}_{c c m}}^{2}\left[R_{0}\right]_{\boldsymbol{\beta}_{r_{c m}} \boldsymbol{\beta}_{c_{c m}}}\left[\boldsymbol{\rho}_{i}\right]_{\boldsymbol{\beta}_{r_{c m}}}+\widehat{\left[R_{i}\right]_{\boldsymbol{\beta}_{r_{d_{i}}} \boldsymbol{\beta}_{d_{d_{i}}}}\left[\boldsymbol{l}_{i}\right]}\right]_{\boldsymbol{\beta}_{d_{d_{i}}}}\left[\dot{\boldsymbol{\omega}_{i}}\right]_{\boldsymbol{\beta}_{c_{d_{i}}}} \\
& \left.+\left[\boldsymbol{l}_{i}\right]_{\boldsymbol{\beta}_{d_{i}}}\left\|\left[\boldsymbol{\omega}_{i}\right]_{\boldsymbol{\beta}_{c_{d_{i}}}}\right\|^{2}\right)=\sum_{i=1}^{n}\left[\boldsymbol{u}_{i}\right]_{\boldsymbol{\beta}_{d_{d_{i}}}}-M_{T} g\left[\boldsymbol{\beta}_{3}\right]_{\boldsymbol{\beta}_{c}} \\
& m_{i}\left(\left[\ddot{\left.\boldsymbol{x}_{0}\right]}-\widehat{\left[R_{0}\right]_{\boldsymbol{\beta}_{r_{c m}} \boldsymbol{\beta}_{c_{c m}}}\left[\boldsymbol{\rho}_{i}\right.}\right]_{\boldsymbol{\beta}_{r_{c m}}}[\dot{\boldsymbol{\omega}}]_{\boldsymbol{\beta}_{c c m}}+\right. \\
& \left.\widehat{[\boldsymbol{\omega}}_{\boldsymbol{\beta}_{c_{c m}}}^{2}\left[R_{0}\right]_{\boldsymbol{\beta}_{r_{c m}} \boldsymbol{\beta}_{c_{c m}}}\left[\boldsymbol{\rho}_{i}\right]_{\boldsymbol{\beta}_{r_{c m}}}+\widehat{\left[R_{i}\right]_{\boldsymbol{\beta}_{r_{d}} \boldsymbol{\beta}_{d_{d i}}}\left[\boldsymbol{l}_{i}\right]}\right]_{\boldsymbol{\beta}_{r_{d_{i}}}}\left[\dot{\boldsymbol{\omega}}_{i}\right]_{\boldsymbol{\beta}_{c_{c m}}}+ \\
& \left.\left[\boldsymbol{l}_{i}\right]_{\boldsymbol{\beta}_{r_{d_{i}}}}\left\|\left[\boldsymbol{\omega}_{i}\right]_{\boldsymbol{\beta}_{c_{d_{i}}}}\right\| \|^{2}\right)=\left[\boldsymbol{u}_{i}\right]_{\boldsymbol{\beta}_{c_{d_{i}}}}-m_{i} g\left[\boldsymbol{\beta}_{3}\right]_{\boldsymbol{\beta}_{c}}
\end{aligned}
$$

iiiEs un sistema de ecuaciones diferenciales bastante horrible...!!! Pero, "algo es algo" como reza el refrán. Las ecuaciones se cotejan aceptablemente bien con las obtenidas por otros autores (Lee, 2014), con algunas discrepancias que requieren ser limadas, y con la gran diferencia de que aquí se conoce a qué corresponde cada uno de los términos, se sabe desde qué sistema de coordenadas se está observando el fenómeno, lo que facilita su interpretación. ¿Pero, qué asumen sobre el fenómeno estas ecuaciones? Esto se puede responder de la siguiente forma,

1. El sistema de coordenadas universal se encuentra fijo, con velocidad nula. La base canónica $\boldsymbol{\beta}_{c}$ genera los elementos de este espacio.
2. Los sistemas de coordenadas locales se mueven con respecto al sistema de coordenadas universal. La base canónica $\boldsymbol{\beta}_{c_{c m}}$ genera los elementos del espacio en el centro de masa de la carga, mientras que la base canónica $\boldsymbol{\beta}_{c_{d_{i}}}$ genera los elementos del espacio en el centro de masa del dron $i$-ésimo.

3. Los ejes de los sistemas de coordenadas locales son paralelos a los del sistema de coordenadas universal, lo que significa que las componentes de un punto en cualquiera de los espacios locales se mantienen igual, si se traslada el vector que representa al punto, en cualquiera de los espacios locales, haciendo coincidir el origen del mismo con el origen del sistema de coordenadas universal. Esto es importantísimo, porque entonces las bases $\boldsymbol{\beta}_{c}, \boldsymbol{\beta}_{c_{c m}} \mathrm{y}$ $\boldsymbol{\beta}_{c_{d_{i}}}$ son las mismas.

4. Cualquier otro sistema de coordenadas local tiene bases no canónicas $\boldsymbol{\beta}_{r_{c m}}$, para el caso del centro de masa de la carga, y $\boldsymbol{\beta}_{r_{d_{i}}}$ para el caso del centro de masa del dron $i$-ésimo, y son una simple rotación que puede ser definida por Euler o Tait-Bryan, u otra rotación válida.

5. Para simplificar el manejo algebraico, se asume que la cuerda del dron $i$ define uno de los ejes del sistema de coordenadas local rotado ubicado en el dron. Asimismo, el vector de posición del punto de amarre desde el sistema de coordenadas local del centro de masa de la carga define uno de los ejes del sistema rotado.

6. Las cuerdas no son elásticas ni flexibles, sino rígidas. Los puntos de amarre con la carga y con los drones semejan bisagras tridimensionales y Figura 3 ilustra cortes longitudinal y transversales de una de las cuerdas y sus amarres, respectivamente.

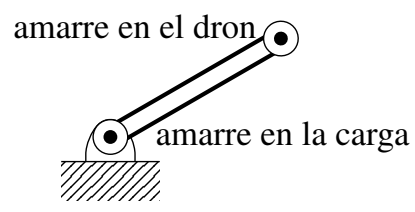

Figura 3: Esquema de amarre dron y carga a través de una cuerda que no es flexible ni elástica, sino totalmente rígida con posibilidades de pivotear en forma tridimensional sobre bisagras localizadas en la carga y el vehículo.

Sólo resta llevar a cabo un rápido "benchmark" de algunos casos límite ya conocidos con los resultados que arrojan las ecuaciones (16), como se indica en las Secciones 5.1 y 5.2.

\subsection{Caso unidimensional}

En este caso, todos los vehículos, al igual que la carga y las cuerdas se mueven en una dimensión, suponiendo que cada uno de ellos y sus correspondientes cuerdas son transparentes a los otros; es decir, que no existen choques o colisiones. Tampoco existen desplazamientos angulares, ni mucho menos velocidades angulares ni rotaciones. Por lo que la única ecuación que describe el fenómeno es la Ecuación (16a) tal como sigue,

$$
M_{T}\left[\ddot{\boldsymbol{x}_{0}}\right]_{\boldsymbol{\beta}_{c}}=\sum_{i=1}^{n}\left[\boldsymbol{u}_{i}\right]_{\boldsymbol{\beta}_{c_{d_{i}}}}-M_{T} g\left[\boldsymbol{\beta}_{3}\right]_{\boldsymbol{\beta}_{c}}
$$


¡Excelente, esto no es otra cosa que la segunda ley de Newton para el caso de un sistema de $n+1$ cuerpos, sujetos a fuerzas gravitacional y de empuje (thrusts)! Coincidiendo con lo que debería esperarse.

\subsection{Caso bidimensional-unidimensional}

Este caso es un poco más difícil de explicar, pues involucra la presencia de una configuración bidimensional con movimiento unidimensional. Los drones, las cuerdas y la carga están localizados en un plano y el movimiento de éste es en una dimensión. Es decir, los drones mueven a la configuración planar en una dirección paralela al plano, tal como lo ilustra Figura 4.

Los sistemas locales de coordenadas pueden rotar solamente alrededor de un eje perpendicular al plano, que pasa por el centro de masa correspondiente a la carga o a cada uno de los drones.

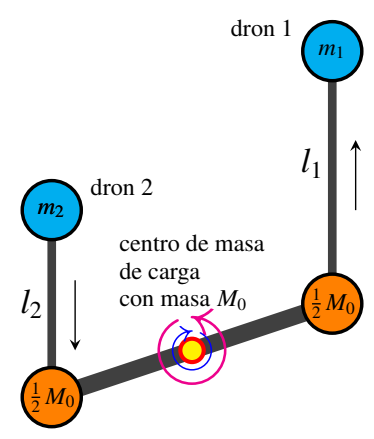

Figura 4: La barra gira alrededor del eje que pasa perpendicularmente al plano atravesando el centro de masa.

Sin embargo, los drones no rotan. Para simplificar aún más la explicación, se asume una carga cilíndrica con diámetro cero y masa distribuida solamente en los extremos, con valor $\frac{1}{2} M_{0}$ en cada caso, y que en cada extremo se enlaza un dron con la carga en forma vertical; es decir, dos drones transportan la carga. Así, el centro de masa de la carga se encuentra a la mitad de la varilla y tiene masa $M_{0}$. En este caso, la Ecuación diferencial (16a) toma la forma siguiente

$$
\begin{array}{r}
M_{T}\left[\ddot{\boldsymbol{x}_{0}}\right]_{\boldsymbol{\beta}_{c}}-m_{1}\left(\frac{L}{2}\left[\begin{array}{c}
\dot{\omega} \sin \theta \\
-\dot{\omega} \cos \theta \\
0
\end{array}\right]_{\boldsymbol{\beta}_{c_{c m}}}+\frac{L}{2}\left[\begin{array}{c}
-\omega^{2} \cos \theta \\
-\omega^{2} \sin \theta \\
0
\end{array}\right]_{\boldsymbol{\beta}_{c c m}}\right)+ \\
+m_{2}\left(\frac{L}{2}\left[\begin{array}{c}
\dot{\omega} \sin \theta \\
-\dot{\omega} \cos \theta \\
0
\end{array}\right]_{\boldsymbol{\beta}_{c c m}}+\frac{L}{2}\left[\begin{array}{c}
-\omega^{2} \cos \theta \\
-\omega^{2} \sin \theta \\
0
\end{array}\right]_{\boldsymbol{\beta}_{c c m}}\right) \\
=\sum_{i=1}^{2}\left[\boldsymbol{u}_{i}\right]_{\boldsymbol{\beta}_{c_{d_{i}}}}-M_{T} g\left[\boldsymbol{\beta}_{3}\right]_{\boldsymbol{\beta}_{c}}
\end{array}
$$

lo que coincide con el resultado que se obtiene al partir de un diagrama de cuerpo libre.

\section{Conclusiones y trabajo futuro}

La especificación del sistema de coordenadas desde el cual se observa el fenómeno es sumamente importante pues, además de facilitar la lectura de las ecuaciones dinámicas del sistema, clarifica la naturaleza del comportamiento complejo del sistema a través de la presencia de transformaciones lineales y/o no lineales, inmersas en las ecuaciones diferenciales y definidas como proyecciones y rotaciones (skew-symmetric matrix) que operan sobre información local que involucra posiciones, velocidades angulares y aceleraciones angulares. Así, el sistema estudiado sugiere un sustento formal al hecho de que transformaciones lineales y no lineales resultan ser una herramienta útil para manipular información y, por consiguiente, hacer patente la presencia de conocimiento, tal y como se define este término aquí. Se está en posibilidad de proporcionar el siguiente ansage ${ }^{1}$ : los resultados sugieren que en términos generales, las ecuaciones que describan procesos en donde se acciona información de cualquier sistema (ojo, ¡cualquier sistema!) deben ser acopladas y con términos que involucren cambios temporales en la información, representados por primeras y segundas derivadas en el tiempo, matrices de transformaciones lineales o no lineales accionando información directamente, o bien sobre cambios temporales de éstas. Las transformaciones "deforman" la información con operaciones tales como traslación, rotación (producto vectorial), escalamiento, reflexión, proyección (observación relativa), etc. ¿Cómo le hacen para ordenar el procesamiento de tal forma que se "observe" como un proceso de razonamiento lógicamente ordenado? No se conoce la respuesta.

Por otro lado, la cuestión de la intencionalidad debe incluirse de alguna manera. Algunos de los autores de este trabajo han obtenido resultados que permiten pensar que los asuntos de intencionalidad se pueden relacionar con formas de controlar el sistema para lograr objetivos planteados (Vargas et al., 2019).

En este caso, las redes neuronales se presentan como las transformaciones apropiadas. Todas esta cuestiones aparecen como trabajo futuro, además de seguir haciendo benchmark de Ecuación (16) con escenarios cuyas soluciones son conocidas, con la idea de validarlas. Se piensa también determinar condiciones para las que el sistema tiene comportamiento caótico, y plantear con ello un formalismo de conocimiento soportado en transformaciones lineales y/o no lineales.

\section{English Summary}

\section{"Knowledge" in a System of Drones Lifting a Load Abstract}

The system of differential equations related to the problem of lifting and transporting a load with a group of drones has a complex coupled structure and a set of linear and nonlinear transformations on several dynamical variables. Fundamentally, the system evolution description shows rotational

\footnotetext{
${ }^{1}$ Anuncio que tal vez sea más que hipótesis, pero que se considera todavía está en etapa de hipótesis
} 
(skew-symmetric matrix), translational (affine), and projective (through a local system of coordinates rotation matrices) transformations; which permits to state the ansage that the "knowledge"2 that a system has appears through the information manipulation by means of linear and/or non-linear transformations. The system of drones lifting and transporting a load is quite interesting because, in addition to what the previous paragraph says, it is useful to introduce the idea of system's intentionality to get a goal, and that this intentionality has an interpretation as transformation. This work introduces the first steps to the analysis of the concept of knowledge in this direction. In this sense, the interpretative twist (based on the idea of lateral thinking) given to the dynamic of the system is unique and promising.

Keywords:

Dynamical System, Drone, Kinetic and Potential Energies, World and Local Coordinates, System of Differential Equations, Center of Mass, Linear and Non-Linear Transformations, Change of Basis

\section{Agradecimientos}

Este trabajo ha sido realizado en parte gracias al apoyo financiero del Consejo Nacional de Ciencia y Tecnología (CONA-
CyT) a través del programa Maestría en Ciencias en Automatización y Control.

\section{Referencias}

de Bono, E., 2009. Penguin Book, New York, USA.

Hoffman, K., Kunze, R., 1971. Prentice Hall, Englewood Cliffs, New Jersey.

Lee, T., 2014. Geometric control of multiple quadrotor uav's transporting a cable-suspended rigid body. arXiv:1403.3684v2 [math.OC] 20 mar 2014 $135,1-10$.

Vargas, E. J., Cansino, J. S., Quesada, E. S. E., Velasco, L. E. R., Carrillo, L. R. G., Lozano, R., 2019. Design and implementation of an artificial neural network wavelet for load transportation with two unmanned aircraft systems. 2019 International Conference on Unmanned Aircraft Systems (ICUAS) accepted for publication.

\footnotetext{
${ }^{2}$ Understood as the capability of actioning information
} 